\title{
Mathematical Analysis of a Diarrhoea Model in the Presence of Vaccination and Treatment Waves with Sensitivity Analysis
}

\section{${ }^{1}$ AKINOLA, EI; ${ }^{* 1}$ AWOYEMI, BE; ${ }^{2}$ OLOPADE, IA; ${ }^{1}$ FALOWO, OD; ${ }^{2}$ AKINWUMI, TO}

\author{
${ }^{* 1}$ College of Agriculture, Engineering, and Science, Bowen University, P. M. B 284. Iwo, Osun State, Nigeria \\ ${ }^{2}$ Department of Mathematics and Computer Science, Elizade University, P. M. B. 002, Ilara-Mokin, Ondo State, Nigeria \\ *Corresponding Author Email: ellybolu@gmail.com
}

\begin{abstract}
In this study, the diarrhoea model is developed based on basic mathematical modelling techniques leading to a system (five compartmental model) of ordinary differential equations (ODEs). Mathematical analysis of the model is then carried out on the uniqueness and existence of the model to know the region where the model is epidemiologically feasible. The equilibrium points of the model and the stability of the disease-free state were also derived by finding the reproduction number. We then progressed to running a global sensitivity analysis on the reproduction number with respect to all the parameters in it, and four (4) parameters were found sensitive. The work was concluded with numerical simulations on Maple 18 using Runge-Kutta method of order four (4) where the values of six (6) parameters present in the model were each varied successively while all other parameters were held constant so as to know the behaviour and effect of the varied parameter on how diarrhoea spreads in the population. The results from the sensitivity analysis and simulations were found to be in sync.
\end{abstract}

\section{DOI: https://dx.doi.org/10.4314/jasem.v25i7.2}

Copyright: Copyright $\left({ }^{\circ} 2021\right.$ Akinola et al. This is an open access article distributed under the Creative Commons Attribution License (CCL), which permits unrestricted use, distribution, and reproduction in any medium, provided the original work is properly cited.

Dates: Received: 10 May 2021; Revised: 28 June 2021; Accepted: 01 July 2021

Keywords: Reproduction number, Equilibrium points, Stability, and Sensitivity analysis.

The growth of some diseases has been on a rise and not enough preventive or treatment measures are being put in place. Some diseases like diarrhoea are even very much neglected because they are considered trivial. Though many researchers have put in much effort in formulating models that could help in eradicating some of these diseases, many of these models have turned out not to be mathematically and epidemiologically well posed. Therefore, this research work was set out to investigate the movement of the disease-diarrhoea throughout a population from susceptible to recovered in all realistic terms, and to evaluate the modelling of infectious diarrhoea in the presence of vaccination, determine the basic reproduction number $R_{0}$, carry out a sensitivity analysis on it, and at the same time stimulate the model so as to draw conclusions that can help medical practitioners/health policy makers know the best control measures to be employed.

\section{MATERIALS AND METHODS}

Model Formulation, Description, and Results Model Assumptions

1. The population is divided into five (5) compartments.

*Corresponding Author Email: ellybolu@gmail.com
2. Rates of transfer between compartments are expressed mathematically as total derivatives with respect to time e.g. $\frac{d S}{d t}$.

3. The mode of transmission of infection being considered is human to human contact of susceptible and infected persons i.e. $\beta S I$

4. Though diarrhoea is more common in kids under the age of five (5) years, the population is considered to be homogeneous and mixed uniformly; there is no special consideration for age, gender, etc.

5. We will consider birth and death rates.

6. Vaccinated population is still susceptible to contracting the disease only in the event that vaccine wanes off; else, they cannot be infected.

Model Analysis: Let $\mathrm{N}$ be the total population of a given region. Therefore, the total population at a given time $\mathrm{t}$ is written as:

$\mathrm{N}(\mathrm{t})=\mathrm{S}(\mathrm{t})+\mathrm{V}(\mathrm{t})+\mathrm{E}(\mathrm{t})+\mathrm{I}(\mathrm{t})+\mathrm{R}(\mathrm{t})$

Where; $\mathrm{S}(\mathrm{t})$ is the number of susceptible persons at time $\mathrm{t}$;

$\mathrm{V}(\mathrm{t})$ is the number of vaccinated persons at time $\mathrm{t}$; $\mathrm{E}$ (t) is the number of exposed persons at time t; 
I (t) is the number of infected persons at time $t$;

$\mathrm{R}(\mathrm{t})$ is the number of recovered persons at time $\mathrm{t}$.

From the description of parameters above we arrived at the following five (5) compartmental model:

$$
\begin{aligned}
& \frac{d S}{d t}=(1-\rho) \lambda-\beta S I+\omega V-\mu S+\theta R=f_{1} \\
& \frac{d V}{d t}=\rho \lambda-\mu V-\omega V=f_{2} \\
& \frac{d E}{d t}=\beta S I-\mu E-\sigma E=f_{3} \\
& \frac{d I}{d t}=\sigma E-\mu I-\delta I-\tau I=f_{4} \\
& \frac{d R}{d t}=\tau I-\mu R-\theta R=f_{5}
\end{aligned}
$$

For this model, the change in each population with respect to time $\mathrm{t}$ is dependent on the parameters described in Table 1 and the transfer of persons from one compartment to another is also clearly represented in Figure 1.

Table 1: Parameter Description

\begin{tabular}{cl} 
& Table 1: Parameter Description \\
\hline Parameters & Description \\
\hline$\lambda$ & Recruitment rate \\
$\beta$ & Contact rate \\
$\rho$ & Vaccination rate \\
$\sigma$ & Infection rate of exposed persons \\
$\mu$ & Natural death rate \\
$\omega$ & Vaccine waning rate \\
$\tau$ & Treatment rate of infected persons \\
$\delta$ & Induced disease death rate \\
$\theta$ & Treatment waves \\
\hline
\end{tabular}

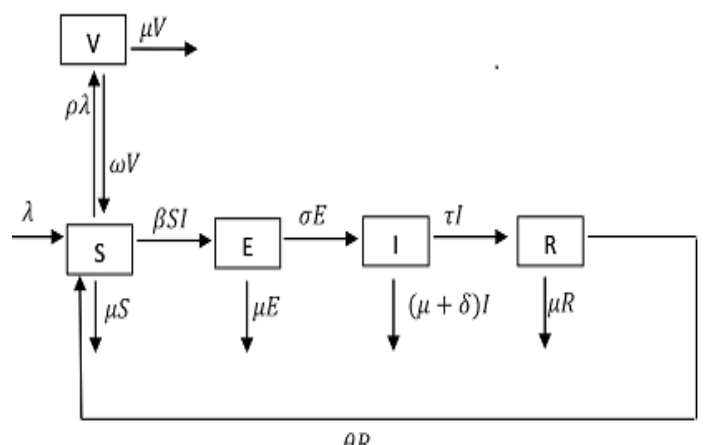

Fig 1: A Schematic (Flow Diagram) Representation of Model

$$
S\left(t_{0}\right)=S_{0}, \quad V\left(t_{0}\right)=V_{0}, \quad E\left(t_{0}\right)=E_{0}, \quad I\left(t_{0}\right)=S_{0}, \quad R\left(t_{0}\right)=R_{0}
$$

Taking the total derivatives of $f_{1}, f_{2}, f_{3}, f_{4}, f_{5}$ with respect to each dependent variables, we have

$$
\begin{array}{lllll}
\frac{d f_{1}}{d S}=-\mu & \frac{d f_{1}}{d V}=\omega & \frac{d f_{1}}{d E}=0 & \frac{d f_{1}}{d I}=0 & \frac{d f_{1}}{d R}=\theta \\
\frac{d f_{2}}{d S}=0 & \frac{d f_{2}}{d V}=-(\mu+\omega) & \frac{d f_{2}}{d E}=0 & \frac{d f_{2}}{d I}=0 & \frac{d f_{2}}{d R}=0 \\
\frac{d f_{3}}{d S}=0 & \frac{d f_{3}}{d V}=0 & \frac{d f_{3}}{d E}=-(\mu+\sigma) & \frac{d f_{3}}{d I}=0 & \frac{d f_{3}}{d R}=0
\end{array}
$$

AKINOLA, EI; AWOYEMI, BE; OLOPADE, IA; FALOWO, OD; AKINWUMI, TO 


$$
\begin{aligned}
& \frac{d f_{4}}{d S}=0 \quad \frac{d f_{4}}{d V}=0 \quad \frac{d f_{4}}{d E}=\sigma \quad \frac{d f_{4}}{d I}=-(\mu+\delta+\tau) \quad \frac{d f_{4}}{d R}=0 \\
& \frac{d f_{5}}{d S}=0 \quad \frac{d f_{5}}{d V}=0 \quad \frac{d f_{5}}{d E}=0 \quad \frac{d f_{5}}{d I}=\tau \quad \frac{d f_{5}}{d R}=-(\mu+\theta)
\end{aligned}
$$

The model satisfies the existence and uniqueness theorems; therefore, a unique solution exists.

Existence of Disease Free Equilibrium: Let $E_{0}$ be the disease free equilibrium. At the disease free equilibrium point, there is no infected person in the We set

$$
\begin{aligned}
& f_{1}=f_{2}=f_{3}=f_{4}=f_{5}=0 \quad(8) \\
& E_{0}=(S, V, E, I, R)=\left(\frac{(1-\rho) \lambda(\mu+\omega)+\omega \rho \lambda}{\mu(\mu+\omega)}, \frac{\rho \lambda}{\mu+\omega}, 0,0,0\right)
\end{aligned}
$$

Existence of Endemic Equilibrium: This is the point where the disease is known to be a part of the population (Van den Driessche and Watmough, 2002). It might have started as an epidemic, but if after a long period of time, there is no change in the population $\mathrm{N}$ and the disease still exists in the population, then we say it is an endemic equilibrium (Van den Driessche and Watmough, 2002). This could mean that the average number of infected persons by one infected person is exactly 1 (Van den Driessche and Watmough, 2002).

Let $E^{*}$ be the endemic equilibrium. For the endemic equilibrium, we work with Equation 8 but

$$
I=E=R=0
$$

And let

$$
\text { Let } E^{*}=\left(S^{*}, V^{*}, E^{*}, I^{*}, R^{*}\right)
$$

Therefore,

$$
\begin{gathered}
1-\rho=C_{1} \\
\mu+\omega=C_{2} \\
\mu+\sigma=C_{3} \\
\mu+\theta=C_{4} \\
\mu+\delta+\tau=C_{5}
\end{gathered}
$$

$$
\begin{gathered}
S^{*}=\frac{C_{3} C_{5}}{\beta \sigma} \\
V^{*}=\frac{\rho \lambda}{C_{2}} \\
E^{*}=\frac{C_{4} C_{5}\left[C_{2} \mu C_{3} C_{5}-C_{1} \lambda C_{2} \beta \sigma-\omega \rho \lambda \beta \sigma\right]}{\beta \sigma C_{2}\left[\sigma \tau \theta-C_{3} C_{4} C_{5}\right]} \\
I^{*}=\frac{C_{4}\left[C_{2} \mu C_{3} C_{5}-C_{1} \lambda C_{2} \beta \sigma-\omega \rho \lambda \beta \sigma\right]}{\beta C_{2}\left[\sigma \tau \theta-C_{3} C_{4} C_{5}\right]} \\
R^{*}=\frac{\tau\left[C_{2} \mu C_{3} C_{5}-C_{1} \lambda C_{2} \beta \sigma-\omega \rho \lambda \sigma\right]}{\beta C_{2}\left[\sigma \tau \theta-C_{3} C_{4} C_{5}\right]}
\end{gathered}
$$

The Basic Reproduction Number $R_{0}$ : The basic reproduction number $R_{0}$ is a significant number that represents how much transferable a disease is, that is it is the average number of infected persons that can be infected by one infectious person during the time of time. This quantity determines whether the infection will spread exponentially, die out, or remain constant depending on if $R_{0}>1, R_{0}<1$ or $R_{0}=1$ (Jones, 2007; Van den Driessche and Watmough, 2002)

By Next Generation Matrix Method (Van den Driessche and Watmough, 2002),

$$
R_{0}=F V^{-1}
$$

Where $F=$ the matrix of compartments containing influx of new infections and $V=$ the matrix of compartments containing other influxes.

$$
\begin{gathered}
F \& V=\left(\begin{array}{ccc}
\frac{\partial f_{3}}{\partial E} & \frac{\partial f_{3}}{\partial I} & \frac{\partial f_{3}}{\partial V} \\
\frac{\partial f_{4}}{\partial E} & \frac{\partial f_{4}}{\partial I} & \frac{\partial f_{4}}{\partial V} \\
\frac{\partial f_{2}}{\partial E} & \frac{\partial f_{2}}{\partial I} & \frac{\partial f_{2}}{\partial V}
\end{array}\right) \\
F=\left(\begin{array}{ccc}
0 & \beta S & 0 \\
0 & 0 & 0 \\
0 & 0 & 0
\end{array}\right) \\
=\left(\begin{array}{ccc}
\mu+\sigma & 0 & 0 \\
-\sigma & \mu+\tau+\delta & 0 \\
0 & 0 & \mu+\omega
\end{array}\right) \\
|V|=(\mu+\sigma)(\mu+\tau+\delta)(\mu+\omega)
\end{gathered}
$$

Since $|V|$ exists, then $V^{-1}$ exists.

We use the Gauss-Jordan or Row Reduction method in finding $V^{-1}, V V^{-1}=I$ where $I$ is the identity matrix. By row reduction, we solve from $(V \mid I)$ to $\left(I \mid V^{-1}\right)$ and then solve for $R_{0}$.

$$
\begin{gathered}
\left(\frac{\sigma \beta S}{(\mu+\sigma)(\mu+\tau+\delta)}-K\right)(-K .-K)=0 \\
K_{3}=\frac{\sigma \beta S}{(\mu+\sigma)(\mu+\tau+\delta)}(\text { Largest eigenvalue })
\end{gathered}
$$


Therefore,

$R_{0}$

$=\sigma \beta \frac{(1-\rho) \lambda(\mu+\omega)+\omega \rho \lambda}{\mu(\mu+\tau+\delta+\sigma)+\sigma(\tau+\delta) \mu(\mu+\omega)}$

Local Stability of Disease Free Equilibrium: For a disease free equilibrium, the local stability implies that if there is a small part of the system (for example, a small number of infected individuals is introduced into the population), then, after some time, the system will return to the disease free equilibrium. However, a big perturbation may be able to drive the system to a different behaviour e.g. to endemic equilibrium. Whereas, if the stability of the disease free equilibrium is global, no matter the size of the perturbation, the disease will not be able to persist in the population (Bello et al., 2019; Obabiyi et al., 2010). These stability points can also be written as local/global asymptotic stability (they are determined at equilibrium points where there is no sign/symptom of change). In this work, we only determined the local stability of the disease free equilibrium which is stable if $R_{0}<1$ and unstable if $R_{0}<1$.

At Disease Free Equilibrium (DFE) where $I=E=R=0$, let the Jacobian matrix of $f_{1}, \ldots, f_{5}=H$

$$
\begin{aligned}
& \frac{\partial f_{1}}{\partial S}=-\mu \quad \frac{\partial f_{1}}{\partial V}=\omega \quad \frac{\partial f_{1}}{\partial E}=0 \quad \frac{\partial f_{1}}{\partial I}=-\beta S \quad \frac{\partial f_{1}}{\partial R}=\theta \\
& \frac{\partial f_{2}}{\partial S}=0 \quad \frac{\partial f_{2}}{\partial V}=-(\mu+\omega) \quad \frac{\partial f_{2}}{\partial E}=0 \quad \frac{\partial f_{2}}{\partial I}=0 \quad \frac{\partial f_{2}}{\partial R}=0 \\
& \frac{\partial f_{3}}{\partial S}=0 \quad \frac{\partial f_{3}}{\partial V}=0 \quad \frac{\partial f_{3}}{\partial E}=-(\mu+\sigma) \quad \frac{\partial f_{3}}{\partial I}=\beta S \quad \frac{\partial f_{3}}{\partial R}=0 \\
& \frac{\partial f_{4}}{\partial S}=0 \quad \frac{\partial f_{4}}{\partial V}=0 \quad \frac{\partial f_{4}}{\partial E}=\sigma \quad \frac{\partial f_{4}}{\partial I}=-(\mu+\delta+\tau) \quad \frac{\partial f_{4}}{\partial R}=0 \\
& \frac{\partial f_{5}}{\partial S}=0 \quad \frac{\partial f_{5}}{\partial V}=0 \quad \frac{\partial f_{5}}{\partial E}=0 \quad \frac{\partial f_{5}}{\partial I}=\tau \quad \frac{\partial f_{5}}{\partial R}=-(\mu+\theta) \\
& \left(\begin{array}{ccccc}
-\mu & \omega & 0 & -\beta S & \theta \\
0 & -(\mu+\omega) & 0 & 0 & 0 \\
0 & 0 & -(\mu+\sigma) & \beta S & 0 \\
0 & 0 & \sigma & -(\mu+\delta+\tau) & 0 \\
0 & 0 & 0 & \tau & -(\mu+\theta)
\end{array}\right)=H
\end{aligned}
$$

Let the polynomial $\begin{gathered}\text { be } \\ K^{2}+K a_{1}+a_{2}=0\end{gathered}$

By Routh-Hurwitz Criterion for a $2 * 2$ system, a polynomial $A=0$ is said to be stable if all $a_{i}$ are positive i.e. $a_{i}>0$

$$
\begin{gathered}
a_{1}=(2 \mu+\sigma+\delta+\tau) \\
a_{2}=\mu(\mu+\sigma+\delta+\tau)+\sigma(\tau+\delta)-\sigma \beta\left(\frac{(1-\rho) \lambda(\mu+\omega)+\omega \rho \lambda}{\mu(\mu+\omega)}\right) \\
\mu(\mu+\sigma+\delta+\tau)+\sigma(\tau+\delta)-\sigma \beta\left(\frac{(1-\rho) \lambda(\mu+\omega)+\omega \rho \lambda}{\mu(\mu+\omega)}\right)>0 \\
\sigma \beta \frac{(1-\rho) \lambda(\mu+\omega)+\omega \rho \lambda}{\mu(\mu+\tau+\delta+\sigma)+\sigma(\tau+\delta) \mu(\mu+\omega)}<1 \\
R_{0}<1
\end{gathered}
$$

Then, DFE is locally asymptotically stable.

Sensitivity Analysis: Sensitivity analysis is the study carried out to determine the input parameters which affect the output of a model most. It is usually or should be run with uncertainty analysis where the output uncertainty of the model is divided to various sources of uncertainty in its inputs (Makinde et al., 2019; Wu et al., 2013).One of the benefits of carrying out sensitivity analysis is that results obtained can be given to experts in the field of the designed model so they can be wary of the parameters that can trigger the model output either by greatly increasing or reducing it.

From Equation 10:

$$
R_{o}=\sigma \beta \frac{(1-\rho) \lambda(\mu+\omega)+\omega \rho \lambda}{\mu(\mu+\tau+\delta+\sigma)+\sigma(\tau+\delta) \mu(\mu+\omega)}
$$

$R_{o}$ will be partially differentiated with respect to 8 parameters: $\beta, \rho, \lambda, \mu, \sigma, \tau, \delta, \omega$.

$$
R_{o}=\frac{\sigma \beta \lambda[\mu(1-\rho)+\omega]}{\mu(\mu+\tau+\delta+\sigma)+\sigma(\tau+\delta) \mu(\mu+\omega)}
$$


Let $X^{R_{0}}$ denote the sensitivity of $R_{o}$, then the sensitivity index $R_{o}$ with respect to any parameter $x$ is

$$
X_{x}^{R_{0}}=\frac{\partial R_{0}}{\partial x} \cdot \frac{x}{R_{0}}
$$

Table 2: Parameter Values and Source of Table 1

\begin{tabular}{lll}
\hline Parameters & Values & Source \\
\hline$\lambda$ & 1000.0 & Assumed \\
$\beta$ & 0.000009 & (Lungu et al., 2018) \\
$\rho$ & 0.5 & (Adewale et al., 2015) \\
$\sigma$ & 0.7 & (Adewale et al., 2015) \\
$\mu$ & 0.012 & (Lungu et al., 2018) \\
$\omega$ & 0.1 & (Adewale et al., 2015) \\
$\tau$ & 0.3 & Assumed \\
$\delta$ & 0.4 & Assumed \\
$\theta$ & 0.01 & Assumed \\
$R_{0}$ & 0.980137466 & Estimated \\
\hline
\end{tabular}

$R_{0}$ in its simplest form is

$$
\begin{aligned}
& R_{o}=\frac{\sigma \beta \lambda[\mu(1-\rho)+\omega]}{\mu(\mu+\tau+\delta+\sigma)+\sigma(\tau+\delta) \mu(\mu+\omega)} \\
& R_{0}=\frac{0.0006678}{0.000681333} \equiv 0.980137466
\end{aligned}
$$

The sensitivity index of each parameter can be seen on Table 3 after inputting the value of each parameter into the differential equations and solving them.

Table 3: Parameter Sensitivity Index

\begin{tabular}{lll}
\hline Parameters & Index & Sensitivity Index \\
\hline$\lambda$ & $X_{\lambda}^{R_{0}}$ & 1 \\
$\beta$ & $X_{\beta}^{R_{0}}$ & 1 \\
$\rho$ & $X_{\rho}^{R_{0}}$ & -0.05660377 \\
$\sigma$ & $X_{\sigma}^{R_{0}}$ & 0.00016923 \\
$\mu$ & $X_{\mu}^{R_{0}}$ & -0.01136718 \\
$\omega$ & $X_{\omega}^{R_{0}}$ & 0.0505501 \\
$\tau$ & $X_{\tau}^{R_{0}}$ & -0.42134815 \\
$\delta$ & $X_{\delta}^{R_{0}}$ & -0.56179754 \\
\hline
\end{tabular}

\section{RESULTS AND DISCUSSIONS}

Numerical simulations: The compartmental model was simulated numerically using Runge-Kutta of the fourth (4th) order on Maple 18. The set of parameters used at different points in the model were used to investigate the effect of vaccine and treatment on the increase/decrease of the different categories of population in relation to the spread of diarrhoea (Adewale et al., 2015; Rachah and Torres, 2017). The values of the parameters $\omega$ (rate at which vaccine wanes off), $\lambda$ (recruitment rate), $\sigma$ (rate at which exposed individuals get infected), $\beta$ (contact rate), $\tau$ (rate at which infected individuals get treated), $\theta$ (rate at which treatment waves) were varied successively.
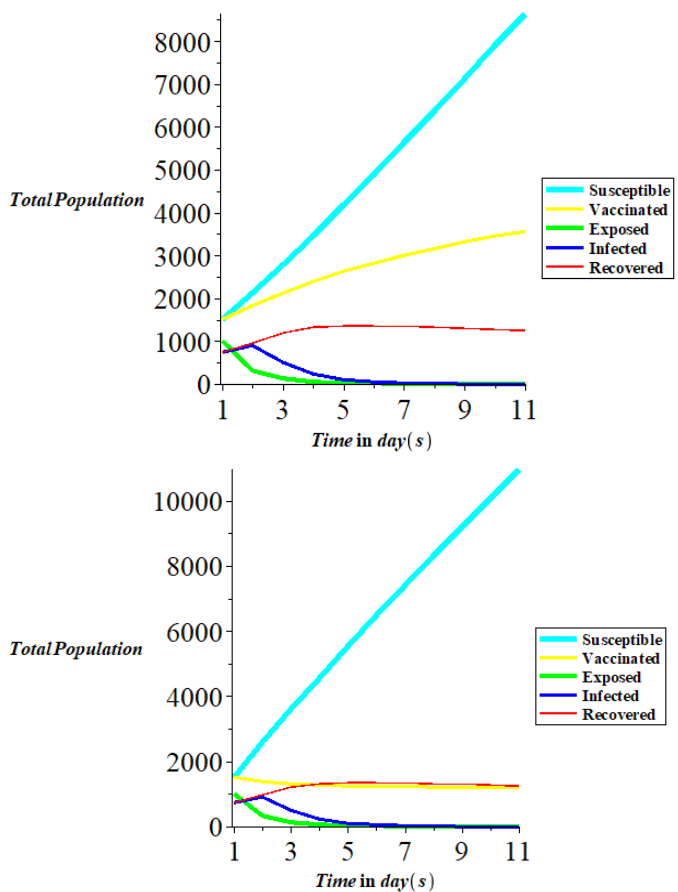

Fig 2: The left panel shows the graph of population against time where $\beta=0.000009, \delta=0.4, \theta=0.01, \mu=0.012, \rho=0.5, \sigma=$ $0.7, \tau=0.3, \omega=0.1, \lambda=1000$ and the right panel is the graph of population against time where $\beta=0.000009, \delta=0.4, \theta=$ $0.01, \mu=0.012, \rho=0.5, \sigma=0.7, \tau=0.3, \omega=0.4, \lambda=1000$.
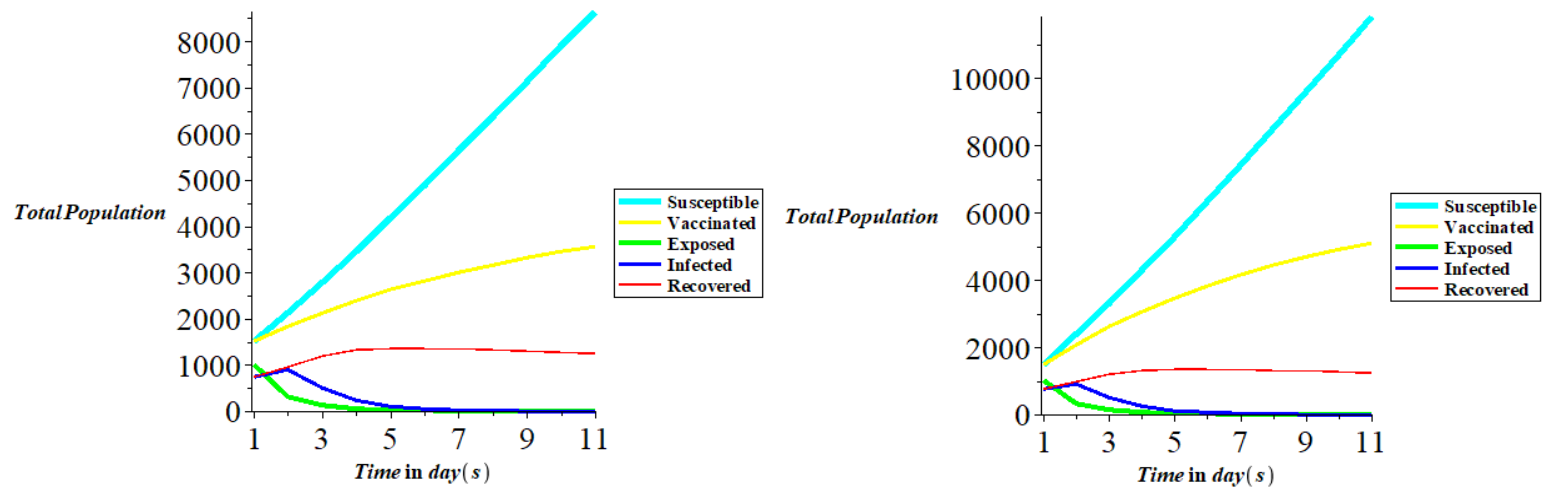

AKINOLA, EI; AWOYEMI, BE; OLOPADE, IA; FALOWO, OD; AKINWUMI, TO 
Fig 3: The left panel shows the graph of population against time where $\beta=0.000009, \delta=0.4, \theta=0.01, \mu=0.012, \rho=0.5, \sigma=0.7, \tau=$ $0.3, \omega=0.1, \lambda=1000$ and the right panel is the graph of population against time where $\beta=0.000009, \delta=0.4, \theta=0.01, \mu=0.012, \rho=$ $0.5, \sigma=0.7, \tau=0.3, \omega=0.1, \lambda=1500$.
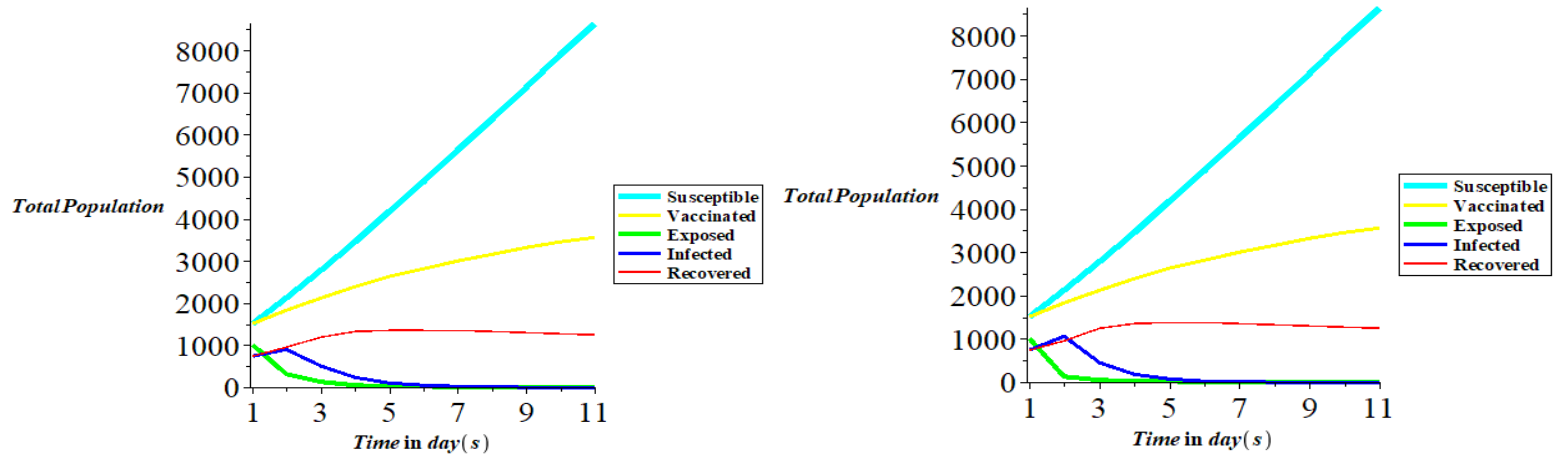

Fig 4: The left panel shows the graph of population against time where $\beta=0.000009, \delta=0.4, \theta=0.01, \mu=0.012, \rho=0.5, \sigma=0.7, \tau=$ $0.3, \omega=0.1, \lambda=1000$ and the right panel is the graph of population against time where $\beta=0.000009, \delta=0 ., \theta=0.01, \mu=0.012, \rho=$ $0.5, \sigma=0.9, \tau=0.3, \omega=0.1, \lambda=1000$
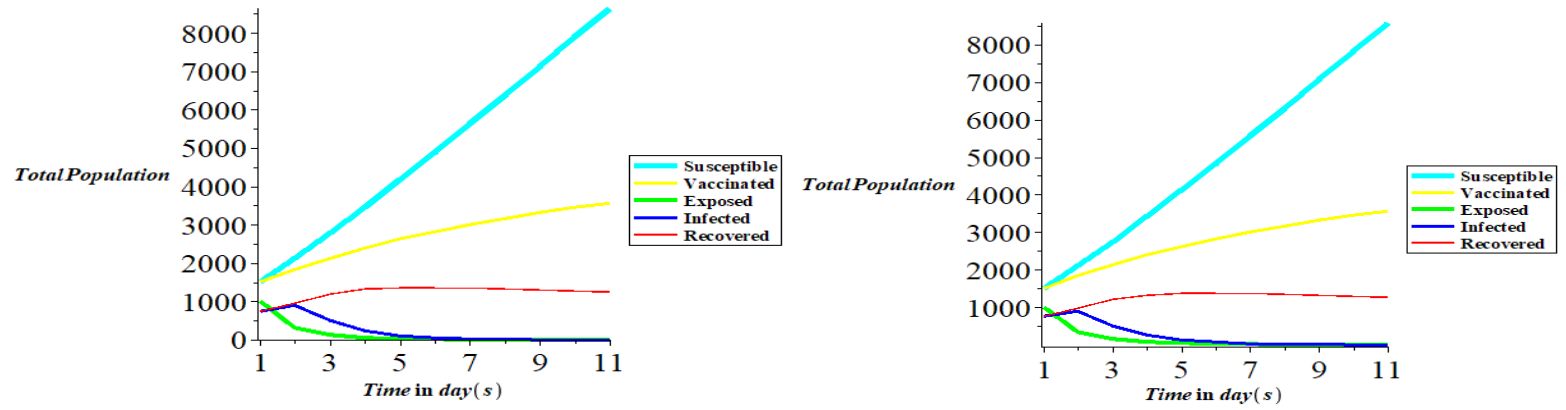

Fig 5: The left panel shows the graph of population against time where $\beta=0.000009, \delta=0.4, \theta=0.01, \mu=0.012, \rho=0.5, \sigma=0.7, \tau=$ $0.3, \omega=0.1, \lambda=1000$ and the right panel is the graph of population against time where $\beta=0.00002, \delta=0.4, \theta=0.01, \mu=0.012, \rho=$ $0.5, \sigma=0.7, \tau=0.3, \omega=0.1, \lambda=1000$.

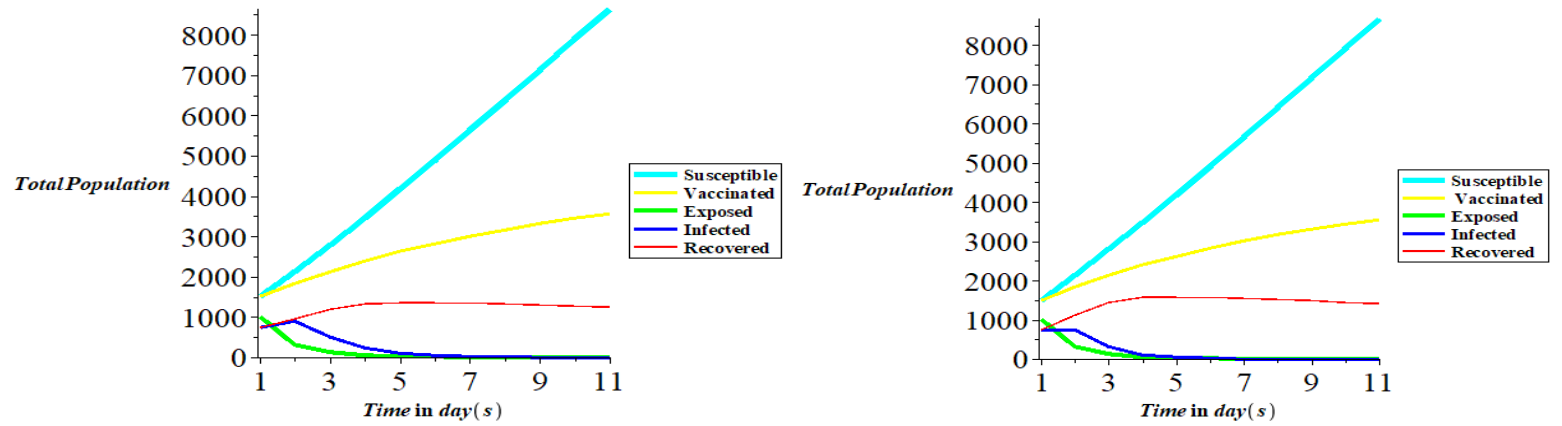

Fig 6: The left panel shows the graph of population against time where $\beta=0.000009, \delta=0.4, \theta=0.01, \mu=0.012, \rho=0.5, \sigma=0.7, \tau=$ $0.3, \omega=0.1, \lambda=1000$ and the right panel is the graph of population against time where $\beta=0.000009, \delta=0.4, \theta=0.01, \mu=0.012, \rho=$ $0.5, \sigma=0.7, \tau=0.5, \omega=0.1, \lambda=1000$ 

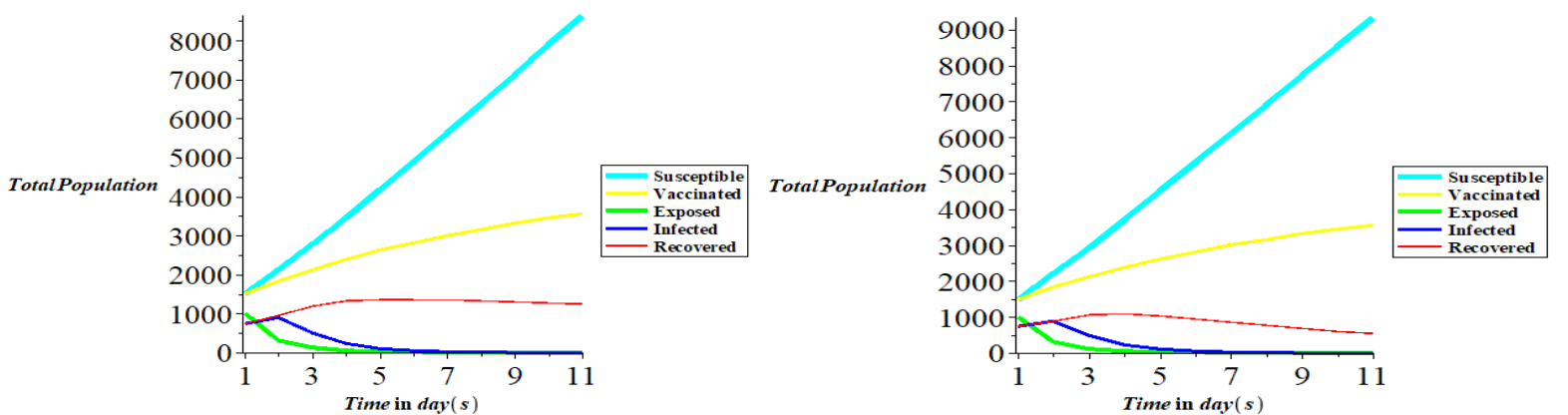

Fig 7: The left panel shows the graph of population against time where $\beta=0.000009, \delta=0.4, \theta=0.01, \mu=0.012, \rho=0.5, \sigma=0.7, \tau=$ $0.3, \omega=0.1, \lambda=1000$ and the right panel is the graph of population against time where $\beta=0.000009, \delta=0.4, \theta=0.1, \mu=0.012, \rho=$ $0.5, \sigma=0.7, \tau=0.3, \omega=0.1, \lambda=1000$.

From figure 2 left panel and right panel, we observe that increase in the rate at which vaccine wanes results in a notable increase in the susceptible population and a significant decrease in the vaccinated population. Figure 3 left panel and right panel show that the higher the influx of the population, the higher the susceptible individuals. From figure 4 left panel and right panel, we observe that the higher the rate at which exposed individuals get infected, the higher the infected population, though the exposed individuals reduce in number. From figure 5 left panel and right panel, it is clear that increase in the contact rate between an infected person and a susceptible person leads to decrease in the susceptible population but a steady increase in the exposed and infected persons. From figure 6 left panel and right panel, we notice that subsequent increase in the treatment rate of infected persons leads to increase in recovered persons and a possible die-out of the disease since the infected and exposed population tend to 0 . From figure 7 left panel and right panel, there is an increase in the rate at which treatment waves result in a sizable increase and decrease in the susceptible and removed population respectively, which is in line with the model that shows us that persons go back to being susceptible once treatment waves.

Conclusion: In conclusion, the strategy that supports vaccines of susceptible individuals should be given a priority in the treatment of diarrhoea, as it reduces the dynamical spread of the disease (diarrhoea) better in the population of the susceptible compared to the population without vaccination. Also, parameters that increase the basic reproduction number $R_{0}$ should be checked by medical practitioners/Policy health makers to guarantee a diarrhoea free environment.

\section{REFERENCES}

Adewale, SO; Olopade, IA; Ajao, SO; Adeniran, GA (2015). Mathematical analysis of diarrhoea in the presence of vaccination. Int. J. Sci. Eng. Res. 6(12): 396-404
Bello, KM; Bello, Y; Abdurhman, S; Akinwande, IN; Kuta, AF (2019). Global stability analysis of the disease-free equilibrium state of a mathematical model of trypanosomiasis. J. Appl. Sci. Environ. Manage. 23: 201-217

Berhe, H; Makinde, O (2019). Modelling the dynamics of direct and pathogens-induced dysentery diarrhoea epidemic with controls. J. Bio. Dyn. 13: 192-217

Castellanos, J; Chen-Charpentier, B; Diaz-Rodriguez, M; Gonzalez-Parra, G; Lozano, DA (2010). Mathematical modeling and characterization of the spread of chikungunya in Colombia. Math. Compt. Appl. 24: 6-22

Castillo-Chvez, C; Song, B (2004). Dynamical models of tuberculosis and their applications. Math. Biosci. Eng. 1: 361-404

Center for Disease Control and Prevention (CDC). Diarrhea: Common illness, global killer, 2015,

https://www.cdc.gov/healthywater/global/diarrheaburden.html

Derricks, WR; Grossman, SI (1976). Elementary Differential Equations with application. Addison Wesley Publications Company, Philippine

Dhar, J; Misra, O; Sinha, S (2014). Stability analysis of sir model with vaccination. Ame.

J. Compt. Appl. Math. 4: 17-23

Gambrah, P; Bonyah, E; Twagirumukiza, G (2019). Mathematical analysis of diarrhoea model with saturated incidence rate. Open J. Math. Sci. 3: 2939

Jones, J (2007). Notes on R0. Department of Anthropological Sciences, Stanford University. 
Li, G; Jin, Z; Wang, W (2006). Global stability of a SEIR epidemic model with constant immigration. Chaos Solitons Fractals. 30: 1012-1019

Li, J; Ma, Z; Zhou, Y (2006). Global analysis of sis epidemic model with a simple vaccination and multiple endemic equilibria. Acta Mathematica Scientia. 26: 83-93

Long, D; Xiang, Z (2011). On the study of an SEIV epidemic model concerning vaccination and vertical transmission. J. Appl. Math. Bioinfo. 1:2130

Lungu, E; Chaturvedi, O; Masupe, S (2017). Epidemic model formulation, analysis and simulation of rotavirus diarrhoea for prevention. Asian. Res. J. Math. 6: 1-14

Lungu, E; Chaturvedi, O; Jeffrey, M; Masupe, S (2018). Rotavirus diarrhea and analysis through epidemic modelling. J. Biomed. Eng. Inform. 4: 21 37

Makinde, O; Berhe, H; Theuri, D (2019). Parameter estimation and sensitivity analysis of dysentery diarrhoea epidemic model. J. Appl. Math. 2019: 113

Momoh, A; Ibrahim, MO; Manga, SB; Uwanta, IJ (2013). Mathematical model for control of measles epidemiology. Ind. J. Pure Appl. Math. 87: 707718

Obabiyi, OS; Nwagwo, A; Raji, II (2010). Stability analysis of a sir epidemic model with constant recruitment. Far East J. Math. Sci. 1: 21-36

Olaniyi, S; Gbadamosi, B; Olopade, I (2013). A nonlinear deterministic model for HIV infection dynamics with optimal control strategy using power series method. J. Nig. Math. Soc. 32: 87-95

Page, N; Leake, C; Murthy, D; Rodin, E (1993). Mathematical modelling: A tool for problem solving in engineering physical, biological and social sciences. J. Oper. Res. Soc. 44: 844-856
Rachah, A; Torres, DFM (2017). Analysis, simulation and optimal control of a seir model for ebola virus with demographic effects. Commun. Fac. Sci. Univ. Ank. Ser. Al Math. Stat. 67(1): 179-197

Rahman, A; Zou, X (2012). Global dynamics of a twostrain disease model with latency and saturating incidence rate. Can. Appl. Math. Quart. 20: 51-73

Roberts, MG; Heesterbeek, J (2003). Mathematical models in epidemiology, Oxford: Eolss

Publishers.

Sun, C; Arino, J; Yang, W, (2010). Global analysis for a general epidemiological model with vaccination and varying population. J. Math. Anal. Appl. 372: 208-223

Thieme, HR (2003). Mathematics in population biology. New Jersey: Princeton University Press.

UNICEF; WHO (2009). Diarrhea: Why children are still dying and what can be done. The United Nations Children's Fund (UNICEF)/ World Health Organization (WHO)

Van den Driessche, P; Watmough, J (2002). Reproduction numbers and sub-threshold endemic equilibria for compartmental models of disease transmission. Math. Biosci. 180(1): 29-48

Wang, C; Xue, X; Lawi, O; Lawrence, O (2015). Modeling the effects of vaccination on rotavirus infection. Advances in Difference Equations

World Health Organization (WHO), Diarrhoeal disease, 2013, https://www.who.int/media/contro/factsheets/fs33 $\underline{0 / \text { en. }}$.

Wu, J; Dhingra, R; Gambhir, M; Remais, JV (2013). Sensitivity analysis of infectious disease models: methods, advances and their application. J. R. Soc. Interface. 10(86):20121018 